\title{
An assessment of the feasibility of sentinel lymph node-guided surgery for gastric cancer
}

\author{
Hiroshi Isozaki ${ }^{1}$, Toshikazu Kimura ${ }^{2}$, Noriaki Tanaka ${ }^{2}$, Katuaki Satoh ${ }^{3}$, Sasau Matsumoto 4 , \\ Motoki Ninomiya ${ }^{5}$, Toshinide Ohsaki ${ }^{6}$, Masanobu Mori $^{7}$, and the Esophagus Gastrointestinal (EGI) Surgical \\ Treatment Study Group* \\ ${ }^{1}$ Oomoto Hospital, 1-1-5 Oomoto, Okayama 700-0924, Japan \\ ${ }^{2}$ Division of Gastroentrological Surgery, Department of Surgery, Okayama University Graduate School of Medicine and Dentistry, \\ Okayama, Japan \\ ${ }^{3}$ Satoh Hospital, Okayama, Japan \\ ${ }^{4}$ Kaneda Hospital, Okayama, Japan \\ ${ }^{5}$ Hiroshima City Hospital, Hiroshima, Japan \\ ${ }^{6}$ Fukuyama National Hospital, Hiroshima, Japan \\ ${ }^{7}$ Onomichi Citizens Hospital, Hiroshima, Japan
}

\begin{abstract}
Background. Sentinel node-guided surgery has received increasing attention in tumor surgery. To ascertain whether sentinel lymph node (SLN)-guided surgery is feasible for gastric cancers $4 \mathrm{~cm}$ or less in size, we conducted a multicenter clinical study.

Methods. One milliliter of isosulfan blue was injected endoscopically into the gastric wall at four sites around a gastric cancer lesion. Approximately $15 \mathrm{~min}$ after the injection of the dye, the surgeons resected (picked-up) the stained blue nodes (defined as SLNs) around the stomach.

Results. SLNs were detected in 140 of 144 patients $(97.2 \%)$. The average number of SLNs was 3.3. In 99 patients with D2 lymph node dissection, the false-negative rate (FNR) was evaluated. In $14 \mathrm{T1}$ patients with pathological positive lymph node metastasis $(\mathrm{pN}(+))$, the FNR was $29 \%$. In 9 T2,3 pN(+) patients, the FNR was $44 \%$. In $\mathrm{T} 1$ patients with $\mathrm{pN}(+)$ but macroscopically normal lymph nodes during surgery (sN0), the FNR was $11 \%(1 / 9)$.

Conclusion. T1 and sN0 patients may be a target group for the study of SLN-guided surgery. A larger multicenter trial should be performed to clarify the application of sentinel node navigation surgery for gastric cancer.
\end{abstract}

Key words Sentinel lymph node - Lymph node metastasis · Gastric cancer · Limited surgery · Isosulfan blue

Offprint requests to: $\mathrm{H}$. Isozaki

Received: September 11, 2003 / Accepted: March 23, 2004

* Members of the EGI group are the First Department of Surgery of Okayama University Hospital; Satoh Hospital; Kaneda Hospital; Hiroshima City Hospital; Fukuyama National Hospital; Onomichi Citizens Hospital; Okayama Saiseikai Hospital; Matsuyama Citizens Hospital; Himeji Central Hospital; Oomoto Hospital; Tsuyama Central Hospital; Kagawa Rohsai Hospital; Matsuda Hospital; and Mihara Red Cross Hospital.

\section{Introduction}

The incidence of early gastric cancer has increased in Japan, with the frequent use of limited operations [1,2] for maintaining the quality of life (QOL) after surgery. When curability is achieved, less extensive surgery (wedge resection without lymph node dissection, for example) results in better QOL after surgery. However, when performing limited surgery, lymph node metastasis is a crucial factor, because it is an important prognostic factor in early gastric cancer $[3,4]$.

Since Morton et al. [5] introduced sentinel lymph node biopsy in patients with melanoma, intraoperative lymphatic mapping and sentinel node-guided surgery have received increasing attention in various types of cancer.

To ascertain whether sentinel lymph node (SLN)guided surgery for gastric cancer is feasible, we conducted a multicenter clinical study.

\section{Patients and methods}

This study included patients with gastric cancer $40 \mathrm{~mm}$ or less in maximum dimension, age 20-80 years, who gave their informed consent. Patients with serious heart disease, pulmonary disease, liver or renal disease, asthma, or allergic history were excluded. Between July 2000 and February 2002, 144 patients were enrolled at 16 centers. The First Department of Surgery of Okayama University Hospital and its affiliated Public Hospitals participated in this study (EGI Surgical Treatment Study Group).

For each patient, after laparotomy and mobilization of the stomach, an endoscope was inserted via the mouth, and $0.25 \mathrm{ml}$ of isosulfan blue (Lympazurin 1\%; 
United States Surgical, Ville St. Laurent, Quebec, Canada) was injected endoscopically into the submucosal layer at four sites (total, $1 \mathrm{ml}$ of isosulfan blue) around the cancer. Approximately $15 \mathrm{~min}$ after the injection of the dye, the surgeons resected only the stained blue nodes around the stomach, and subsequently gastrectomy with lymphadenectomy was performed. Only the stained nodes were defined as SLNs. Blue nodes detected during lymphadenectomy or after the gastrectomy were not defined as sentinel nodes.

Assessment of lymph node metastasis during surgery (sN, including preoperative clinical findings) was done by surgeons according to gross findings or palpation of the perigastric lymph nodes; also, assessment was done in the fresh specimens. Size (enlarged), shape (round), and firmness (firm) of the lymph nodes were evaluated. However, no strict definition for macroscopic positive lymph node metastasis was made in the protocol of this study.

Histological examination of the lymph nodes was performed by hematoxylin-eosin staining of one section of each node.

The false-negative rate (FNR) by SLN biopsy was defined as: Number of patients with pathological lymph node metastasis $(\mathrm{pN}(+))$ and with negative metastasis in SLN / Number of $\mathrm{pN}(+)$ patients.

This report uses the terminology of the Japanese classification of gastric carcinoma [6].

\section{Results}

SLNs (blue nodes) were detected in 140 of the 144 patients $(97.2 \%)$.

The average number of SLNs was 3.3 per positive patient (range, 1-9). The average number of removed lymph nodes per patient was 26 (range, 10-69).

Table 1 gives the clinicopathological details of the 140 patients in whom SLNs were detected.

Table 2 shows the distribution of SLNs according to the Japanese classification of gastric carcinoma, In 76\% of the patients, SLNs were located in compartment 1 only. The first compartment was negative in $2 \%$ of the patients with SLNs.

In 99 patients with D2 node dissection, the falsenegative rate (FNR) was evaluated (Table 3 ). It ranged between $11 \%$ and $44 \%$, being lowest in $\mathrm{T} 1$ patients with $\mathrm{pN}(+)$ but macroscopically normal lymph nodes during surgery.

Figure 1 shows the relation between the location of SLNs (stained lymph nodes) and the location of metastatic nodes in the $14 \mathrm{~T} 1$ patients with pathological lymph node metastasis and D2 dissection.

In seven patients, SLNs were detected in one node station only. In four of them, SLNs were detected at the
Table 1. Clinicopathological features of 140 patients with sentinel lymph node (SLN) detection

\begin{tabular}{lc}
\hline & No. of patients \\
\hline Depth of tumor invasion & \\
T1 & $125(90 \%)$ \\
T2 & $13(9 \%)$ \\
T3 & $2(1 \%)$ \\
Pathological lymph node metastasis & \\
pN0 & $115(82 \%)$ \\
pN1 & $21(15 \%)$ \\
pN2 & $3(2 \%)$ \\
pN3 & $1(1 \%)$ \\
Macroscopic lymph node staging during surgery & \\
sN0 & $128(92 \%)$ \\
sN1 & $10(7 \%)$ \\
sN2 & $2(1 \%)$ \\
Lymph node dissection & \\
D0 & $12(9 \%)$ \\
D1 & $29(21 \%)$ \\
D2 & $97(69 \%)$ \\
D3 & $2(1 \%)$ \\
\hline
\end{tabular}

Table 2. Distribution of the sentinel lymph nodes (SLNs)

\begin{tabular}{lc}
\hline Location of SLN $^{\mathrm{a}}$ & No. of patients \\
\hline Compartment 1 only & $107(76 \%)$ \\
Compartments 1 and 2 & $29(21 \%)$ \\
Compartment 2 only & $3(2 \%)$ \\
Compartments 1 and 3 & $1(1 \%)$ \\
\hline
\end{tabular}

${ }^{a}$ Regional lymph node compartments according to the Japanese Classification of gastric carcinoma [6]

lesser curvature side, and all four had metastatic nodes at this side; one patient also had a metastatic node at the left gastric artery. However, in three patients with sentinel nodes at the greater curvature, two patients with sentinel nodes in station $4 \mathrm{~d}$ also had a lymph node metastasis at the lesser curvature.

Figure 2 is a diagram of a false-negative case in a patient with $\mathrm{T} 1$ tumor. The tumor was located at the greater curvature, and SLNs at the greater curvature were normal, but a perigastric node, $1.5 \mathrm{~mm}$ in diameter at the lesser curvature was metastatic. Moreover, in this patient, immunohistochemistry revealed micrometastasis along the common hepatic artery.

Figure 3 shows the distribution of false-negative cases by SLN biopsy according to the number of cases in each hospital. False negativity tended to occur early in each series; however, there was no statistically significant difference between numbers of early and late falsenegative cases. 
Table 3. False-negative rate (FNR) in 23 patients with pathological lymph node metastasis out of 99 patients with D2 lymph node dissection evaluated for FNR

\begin{tabular}{|c|c|c|c|}
\hline & Metastasis in SLN & No metastasis in SLN & FNR $(\%)$ \\
\hline T1; All 14 patients & 10 & 4 & 29 \\
\hline $\mathrm{T} 1 \mathrm{pN}(+) \mathrm{sN}(-) ; 9$ Patients ${ }^{\mathrm{a}}$ & 8 & 1 & 11 \\
\hline $\mathrm{T} 2,3 ; 9$ Patients & 5 & 4 & 44 \\
\hline $\mathrm{T} 2,3 \mathrm{pN}(+) \mathrm{sN}(-) ; 5$ Patients $^{\mathrm{a}}$ & 3 & 2 & 40 \\
\hline
\end{tabular}

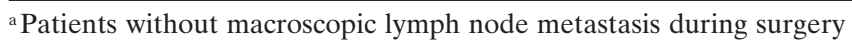

\begin{tabular}{llll}
\hline Location of SLNs & $\begin{array}{l}\text { Number of } \\
\text { patients }\end{array}$ & $\begin{array}{l}\text { Location of LNs } \\
\text { with metastasis }\end{array}$ & $\begin{array}{l}\text { Number of } \\
\text { patients }\end{array}$ \\
\hline
\end{tabular}

$\begin{array}{llll}\text { Lesser curvature only (LC) } & 4 \longrightarrow & \text { LC } & 4 \\ \text { Greater curvature only (GC) } 3 \longrightarrow & \text { GC } & 1 \\ \text { GC and LC } & 1 \\ \text { LC } & \end{array}$

$\begin{array}{lll}\text { LC and GC } & \longrightarrow & \text { LC } \\ \text { LC and GC } & 4 \\ \text { GC } & 1 \\ \end{array}$

Fig. 1. Relation between the locations of sentinel lymph nodes (SLNS) (stained nodes) and of metastatic nodes in $14 \mathrm{~T} 1$ patients with pathological lymph node metastasis $(\mathrm{pN}(+))$ and $\mathrm{D} 2$ dissection. Asterisks, metastasis in locations differing from SLNs

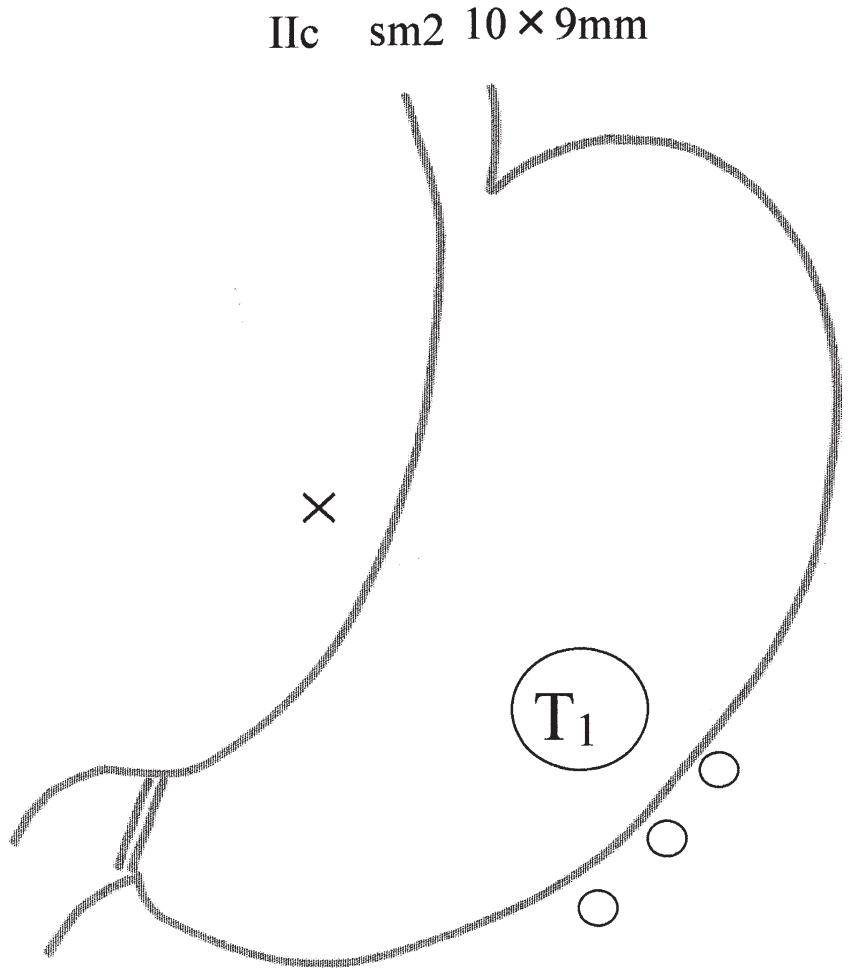

Fig. 2. False-negative $T 1$ case with node metastasis outside the station containing SLNs. The specimen contained 30 nodes (there were 3 SLNs and another ([non-SNL] metastatic node). Circles, SLNs without metastasis; cross, non-SLN with metastasis; $s m$, submucosal

\section{Discussion}

Sentinel lymph nodes (SLNs), the first draining nodes around a tumor, are expected to be the first nodes which contain metastasis. Intraoperative lymphatic mapping and sentinel lymphadenectomy are currently used to guide the use of complete lymphadenectomy in patients with positive sentinel nodes in melanoma or breast cancer $[7,8]$.

The application of sentinel node tactics in gastrointestinal malignancy and gastric surgery has been controversial [9-11]. In gastric cancer, Hiratsuka et al. [12] reported that, using indocyanine green, the sensitivity for detecting node metastasis by the SLN technique was $100 \%$ for $\mathrm{T} 1$ tumors and $88 \%$ for T2 tumors. However, few patients were studied. Miwa et al. [13] used patent blue and found the sensitivity of SLN for T1 gastric cancer to be $89 \%(31 / 35)$. All their false-negative cases had macroscopic metastasis at surgery, so the sensitivity for SLN in sN0 patients was $100 \%$. Their method to detect SLN was to dissect the stained lymphatic region (lymphatic basin ${ }^{13}$ ), and then search for SLNs on a back table during surgery. Kitagawa et al. [14], using technetium-99m-labeled tin colloid solution, found the SLN method to be positive in 22 of 24 (91.7\%) T1-2, cN0 (evaluated clinically as N0), M0 patients with node metastasis. 


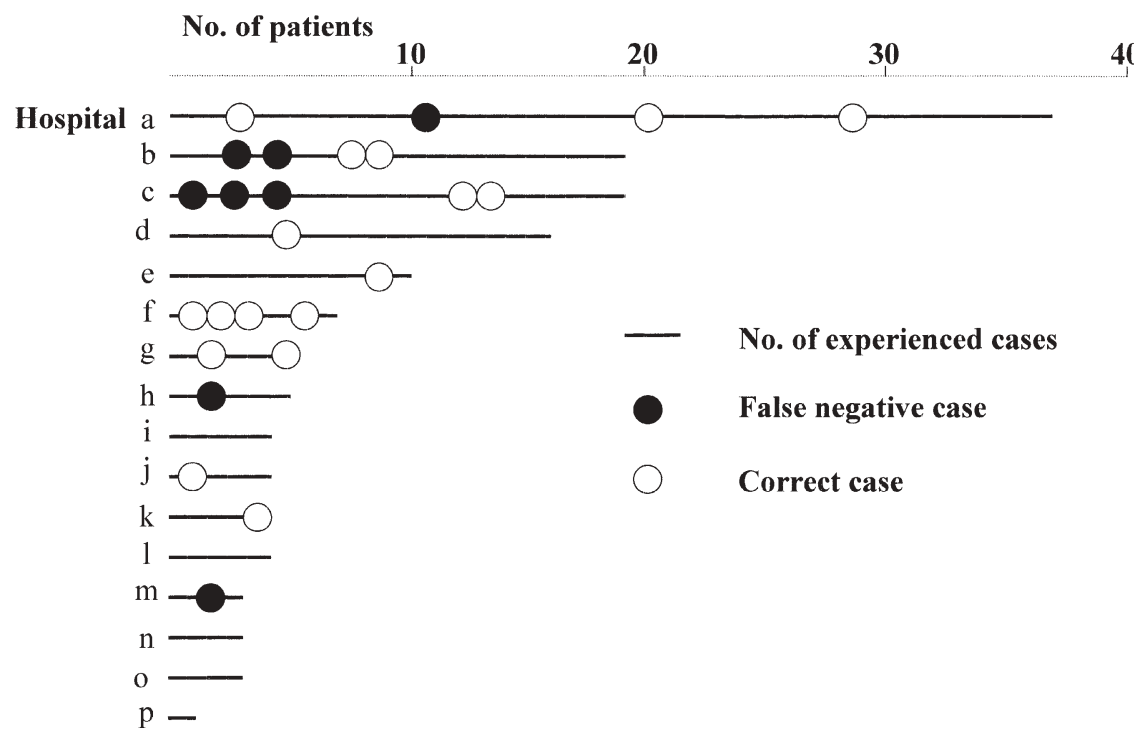

Fig. 3. Distribution of false-negative cases by SLN biopsy according to the number of cases experienced at each hospital (in 140 patients with SLN detection)
When we started this multicenter study, we considered the following items. Firstly, gastric cancer is very common in Japan and is treated surgically at a large number of hospitals. Consequently, these hospitals should be included in multicenter trials, even if they treat few patients. Secondly, in Japan, it is difficult to use radioactive substances at smaller hospitals; consequently, we used a dye (isosulfan blue). Concerning the administration of the tracer, Hiratsuka et al. [12] injected the indocyanine green subserosally via the serosa. Miwa et al. [13] and Kitagawa et al. [14] injected the dye or radioactive colloid into the submucosa endoscopically. We injected the dye endoscopically according to Miwa et al. [13], because many T1 tumors were difficult to palpate.

In our study, SLNs were detected in $97.2 \%$ patients, and the average number of SLNs per patient was 3.3. These findings were comparable to single-center experiences, even though Miwa et al. [13] found an average number of SLNs of 6. Our false-negative rate of SLN for the detection of node metastasis was higher than in previous studies. However, when limited to T1 tumors without macroscopic metastasis during surgery, but with pathological positive metastasis, the sensitivity of SLN for lymph node metastasis was comparable with that reported from single centers.

This study indicated that $\mathrm{T} 1$ and sN0 patients may be a target group for SLN-guided surgery. The purpose of the SLN biopsy is to avoid extensive node dissection. However, lymphatic flow from the stomach spreads in various directions, and is more complex than that of the breast or extremities. To avoid false-negative cases, multi-directional lymphatic flow should be considered in the application of the SLN technique. Moreover, there is a possible problem of micrometastasis in addi- tion to false-negative cases. Consequently, for patients with T1 SLN (-) tumors which may have node metastases, complete avoidance of node dissection is not yet recommended.

For T2 tumors, the false-negative rate of SLN was high in this study, suggesting that SLN-guided surgery is not applicable. In Japan, most surgeons perform D2 lymph node dissection for these tumors because of the high rate of lymph node metastasis.

In conclusion, $\mathrm{T} 1$ and $\mathrm{sN} 0$ patients may be a target group for future studies on SLN-guided surgery. A larger multicenter trial with a working definition of $\mathrm{sN}(+)$ and a learning period could possibly clarify the role of sentinel node navigation surgery in that situation.

\section{References}

1. Isozaki H, Okajima K, Nomura E, Ichinona T, Fujii K, Izumi N, et al. Postoperative evaluation of pylorus-preserving gastrectomy for early gastric cancer. Br J Surg 1996;83:266-9.

2. Furukawa $\mathrm{H}$, Hiratsuka $\mathrm{M}$, Imaoka $\mathrm{S}$, Ishikawa $\mathrm{O}$, Kabuto $\mathrm{T}$, Sasaki T, et al. Phase II study of limited surgery for early gastric cancer: segmental gastric resection. Ann Surg Oncol 1999;6:16670.

3. Kim JP, Hur YS, Yang HK. Lymph node metastasis as a significant prognostic factor in early gastric cancer: analysis of 1136 early gastric cancers. Ann Surg Oncol 1995;2:308-13.

4. Isozaki H, Tanaka N, Okajima K. General and specific prognostic factors of early gastric carcinoma treated with curative surgery. Hepatogastroenterology 1999;46:1800-8.

5. Morton DL, Wen DR, Wong JH, Economous JS, Cagle LA, Storm FK, et al. Technical details of intraoperative lymphatic mapping for early stage melanoma. Arch Surg 1992;127:3929.

6. Nishi M, Omori Y, Miwa K, editors. Japanese Research Society of Gastric Cancer. Japanese classification of gastric carcinoma, 1st Ed. Tokyo: Kanehara; 1995. p 1-31. 
7. Morton DL, Thompson JF, Essner R, Elashoff R, Stern SL, Nieweg OE, et al. Multicenter Selective Lymphadenectomy Trial Group. Validation of the accuracy of intraoperative lymphatic mapping and sentinel lymphadenectomy for early-stage melanoma. A multicenter trial. Ann Surg 1999;230:453-65.

8. Morrow M, Rademaker AW, Bethke KP, Talamonti MS, Dawes LG, Clauson J, et al. Learning sentinel node biopsy: results of a prospective randomized trial of two techniques. Surgery 1999;126: 714-22.

9. Joosten JJA, Strobbbe LJA, Wauters CAP, Pruszczynski M, Wobbes Th, Ruers TJA. Intraoperative lymphatic mapping and the sentinel node concept in colorectal carcinoma. Br J Surg 1999; 86:482-6.

10. Chin PL, Medeiros J, Schwarz RE. Use of the sentinel node to determine metastases of gastrointestinal malignancies: a word of caution. J Surg Oncol 1999;71:239-42.
11. Palaia R, Cremona F, Delrio P, Izzo F, Ruffolo F, Parisi V. Sentinel node biopsy in gastric cancer. J Chemother 1999;11:2301.

12. Hiratsuka M, Miyashiro I, Ishikawa O, Furukawa H, Motomura $\mathrm{K}$, Ohigashi $\mathrm{H}$, et al. Application of sentinel node biopsy to gastric cancer surgery. Surgery 2001;129:335-40.

13. Miwa K, Kinami S, Taniguchi K, Fushida S, Fujimura T, Nonomura A. Mapping sentinel node in patients with early-stage gastric carcinoma. Br J Surg 2003;90:178-82.

14. Kitagawa Y, Fujii H, Mukai M, Kubota T, Otani Y, Kitajima M. Radio-guided sentinel node detection for gastric cancer. Br J Surg 2002;89:604-8 